\title{
An Automatic Offsetting Method of Composite Surfaces
}

\author{
Hiroyuki Kawabata*, Junji Ishida** \\ * TOYOTA MOTOR CORPORATION, 1, Toyota-cho, Toyota, Aichi, 471-8571, Japan \\ ** TOYOTA Soft Engineering Inc., 2-3-31, Sakae, Naka-ku, Nagoya, Aichi, 460-0008, Japan
}

Key words: offset, composite surfaces, global modification, calculation of surface intersection, topological relation

Abstract: In the process of modelling the inner surface of resin parts for an automobile, we have usually offset competent surfaces of the outer model and relied on tedious joining operations to guarantee the integrity of the result.

Consequently, considerable amount of manpower has been required for this process.

It is well known that conventional methods based on repetitive offset of component surfaces have weakness of causing geometric deviation from neighbouring surfaces. We believe that the technique of offsetting a whole composite surface, which consists of geometric surfaces representing the object shape and their topological relationship, is highly effective to overcome the weakness. Our investigation, however, shows that no commercial CAD system using this technique has reached a practically satisfactory level in view of numerical stability.

This report presents our own technique that we have developed to overcome the problem and meet the practical requirements. It includes global modification and recognition of topological changes in the offset composite surface. We believe that it has realized automatic modelling of inner surface for specific parts.

\section{INTRODUCTION}

Modelling of complicated inner surfaces of resin parts such as a bumper for an automobile has remained unsolved and we had to offset the outer 
surfaces repetitively surface by surface and underwent tedious joining operations using CAD. Particularly, for resin parts such as bumper, etc., variable offset is an important requirement from the viewpoints of improved strength for the mounting flanges and openings, material cost reduction, improvement of fluidity and so forth. Thus far we failed to have an effective means and have been obliged to concentrate CAD operation man-hours.

Principally speaking, repetitive offset of component surfaces requires excessive manpower for adjusting geometric deviation of surfaces after the offsetting. Consequently, it is thought effective to make an overall offset of an object shape by expressing it with geometric and topological information (relations of connectivity between points, lines and surfaces, hereinafter referred to as the composite surfaces). On the basis of the fundamental studies $^{(1)(2)}$ on this method, commercial CAD systems have lately begun to materialize this idea. Our investigation shows, however, that these systems have not reached a practical level of application because of the following problems (see Table 1):

a) Variable offset cannot be handled.

b) If source surfaces are in continuity of tangent planes (continuity of the

first derivatives), crack on the offset surfaces, if any, cannot be avoidable or repaired.

c) The technique is not fully capable of handling cases where the topological relation changes before and after the offset.

Problem a) means the lack of an essential function for the modelling of resin parts and problem b) is unavoidable as long as error is present in the connection of the source surfaces. Problem c) occurs when the offset exceeds the radius of curvature or the width of a surface. This is the problem that always accompanies the modelling of resin parts such as bumpers.

This report presents our own overall offset algorithm which we have developed for practical application by overcoming the shortcomings of conventional method, reducing the surface modelling manpower and subsequently realizing the shorter lead time.

\section{PROBLEMS AND COUNTERMEASURES}

Table 1 indicates the measures taken for solving the shortcomings of the conventional method as found in the commercial CAD systems. 

Table 1 Problems of Overall Offset of Composite Surfaces and
Countermeasures

\begin{tabular}{|c|c|c|c|c|c|}
\hline \multirow{2}{*}{$\#$} & \multirow{2}{*}{ Problem } & \multirow{2}{*}{ Reason } & \multirow{2}{*}{ Countermeasure } & \multicolumn{2}{|c|}{ Actual example } \\
\hline & & & & Source surface & Offset surface \\
\hline 1 & $\begin{array}{l}\text { Varible ortiex cannot } \\
\text { be haodied. }\end{array}$ & \multirow{2}{*}{ 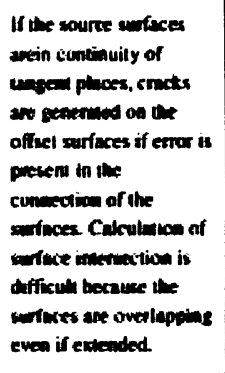 } & \multirow{2}{*}{ 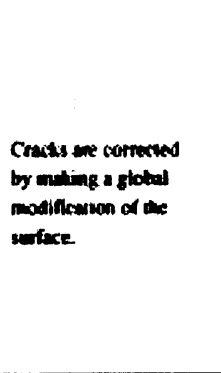 } & & \\
\hline 2 & 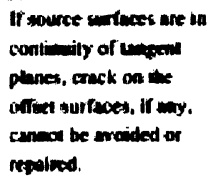 & & & & \\
\hline \multirow{6}{*}{\multicolumn{2}{|c|}{ 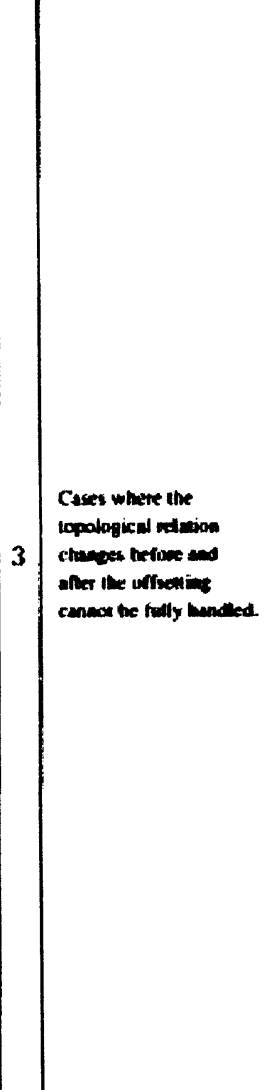 }} & 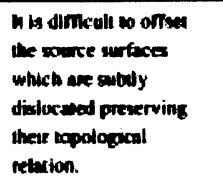 & 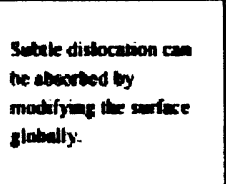 & & \\
\hline & & \multirow{5}{*}{ 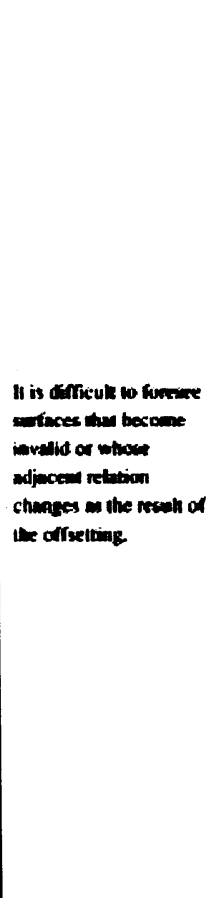 } & 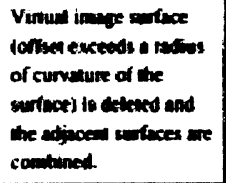 & & \\
\hline & & & 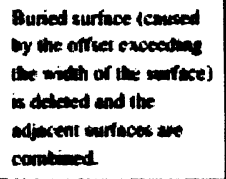 & & \\
\hline & & & 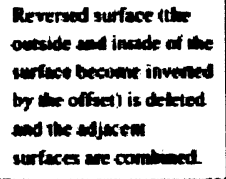 & & \\
\hline & & & 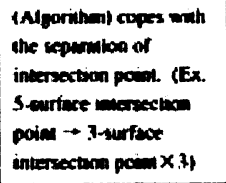 & & \\
\hline & & & 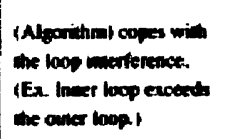 & & \\
\hline
\end{tabular}


Fig. 1 shows the processing flow that incorporates all of the measures indicated in Table 1.

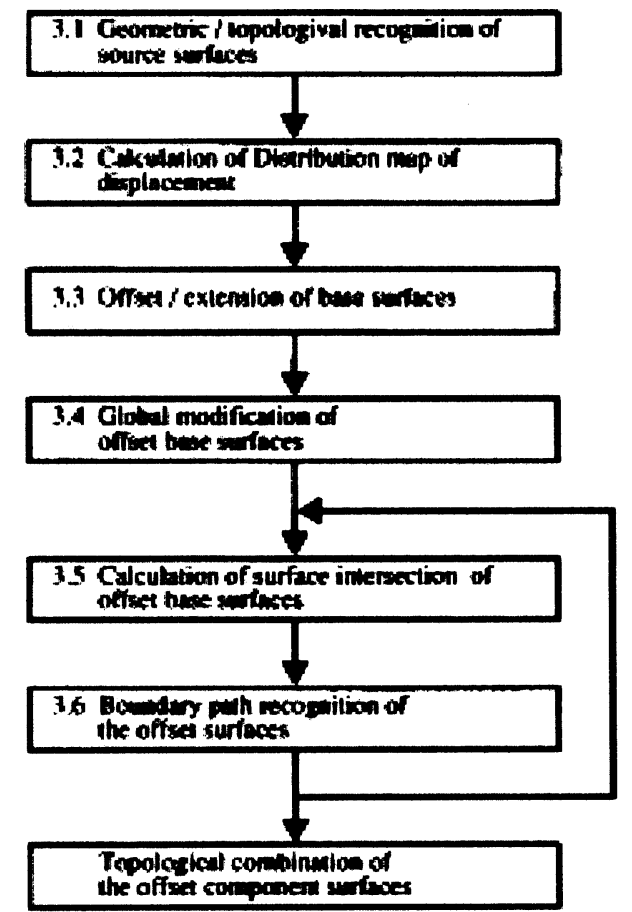

Fig.1 Processing Flow

\subsection{Geometric / Topological Recognition of Source Surfaces}

Folding angles of respective component surfaces are detected from the geometric relation of the source surface and the direction of offset to judge whether the surfaces are of convex, concave or AF (Almost Flat) combination. The AF combination refers to a combination of surfaces that can be regarded as being approximately in continuity of tangent planes. An offset base surface is an object global modification. The offset base surface refers to a surface expressed with its geometric information only. A surface expressed with its base surface and its boundary information is called a component (trimmed) surface. 
If a surface combination is judged as convex, the length of extension of the offset base surface is calculated from the folding angle, the offset value and the distance from the component surface boundary to the base surface boundary.

The sequential recognition of the global modification of a surface and the boundary path are made from the topological relation of the source surface.

\subsection{Calculation of Distribution Map of Displacement}

When a composite surface whose offset varies from $\mathrm{d} 0$ to $\mathrm{d} 1 \mathrm{is}$ laid out as shown in Fig.2, distances $\mathrm{r} 0$ and $\mathrm{r} 1$ from a vertex $\mathrm{V}$ of the component source surface to the constant-offset boundary edge of $\mathrm{d} 0$ and to that of $\mathrm{d} 1$ are obtained respectively. Then offset $d 0+d(r)$ of the vertex $O V$ is obtained by defining a distribution map of displacement $\mathrm{d}(\mathrm{r})$ using a cubic polynomial. These offset values of $\mathrm{OV}$ of each source surface are averaged to determine its constant-offset value.

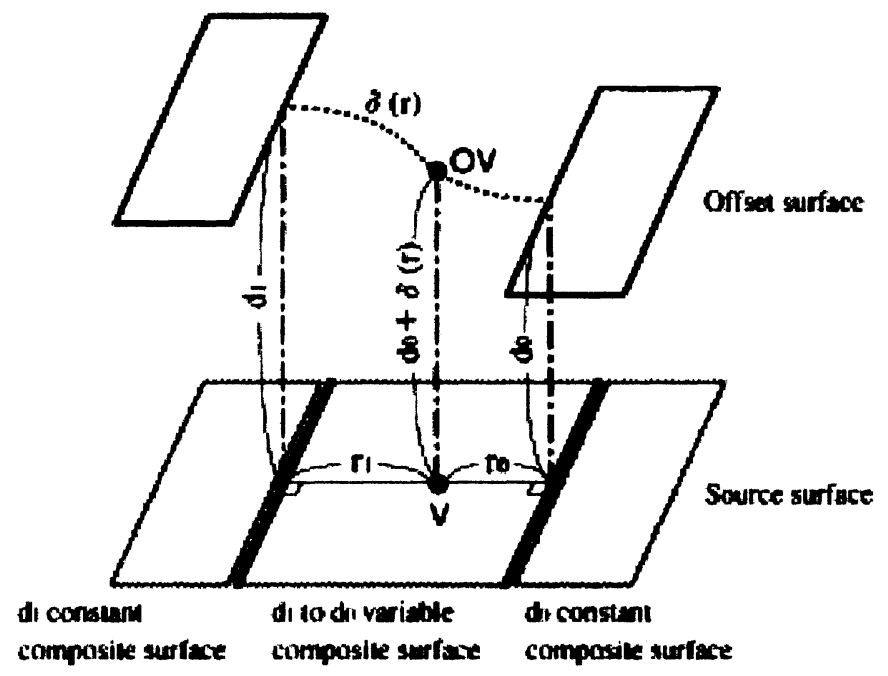

Fig.2 Variable Layout of the Vertex

\subsection{Base Surface Offset / Extension}

The base surface is constant-offset and if the result is a virtual image, the offset surface is deleted. Then the offset base surface is extended according to the extension obtained from the preceding item 3.1. 


\subsection{Global Modification of Offset Base Surface}

An offset surface that corresponds to the AF combination boundary in the source surface as detected in the preceding item 3.1 is globally modified.

Global modification refers to obtaining OS' $(u, v)$ by deforming the offset base surface OS(u,v) as shown in Fig. 3 through the following equation which uses the boundary edges and their cross-boundary derivatives of the surfaces to be combined as its constraint conditions:

$$
\mathrm{OS}^{\prime}(\mathrm{u}, \mathrm{v})=\mathrm{OS}(\mathrm{u}, \mathrm{v})+\left(\mathrm{OG}^{\prime}(\mathrm{u}, \mathrm{v})-\mathrm{OG}(\mathrm{u}, \mathrm{v})\right)
$$

Where:

OG(u,v) and OG'(u,v) represent the Gordon surfaces interpolated from only the boundary edges and their cross derivatives of OS(u,v) and those of $\mathrm{OS}^{\prime}(\mathrm{u}, \mathrm{v})$ respectively.

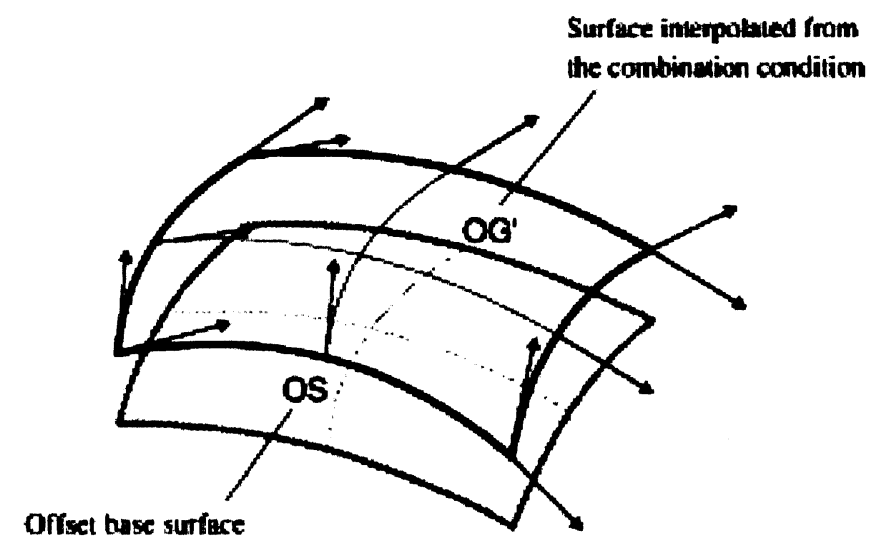

Fig. 3 Constraint Conditions of Global Modification

The global modification is applied to correct cracks and misalignment between the constant/variable offset surfaces. However, once combined surfaces become cracked again as become cracked as shown in Fig.4(a) unless the global modification is made in an appropriate sequence. To prevent this, a deformation sequence has to be established in advance so that a condition that the surfaces to be combined never be deformed again may be satisfied as illustrated in Fig.4(b). This is the meaning of the recognition of the sequence of a global modification. 


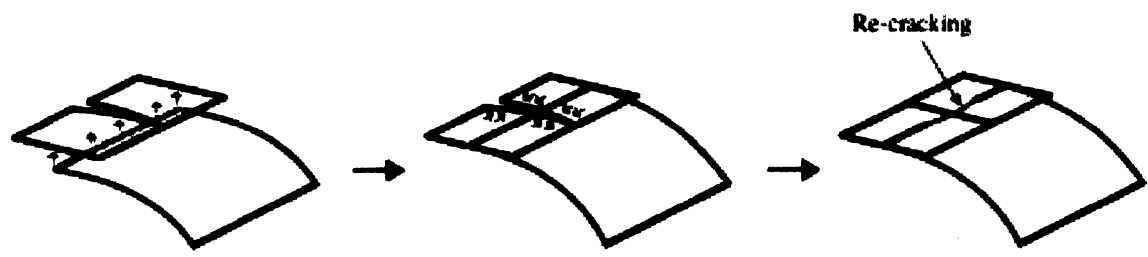

(a) Example of inappropratis sequence of global modilicalion

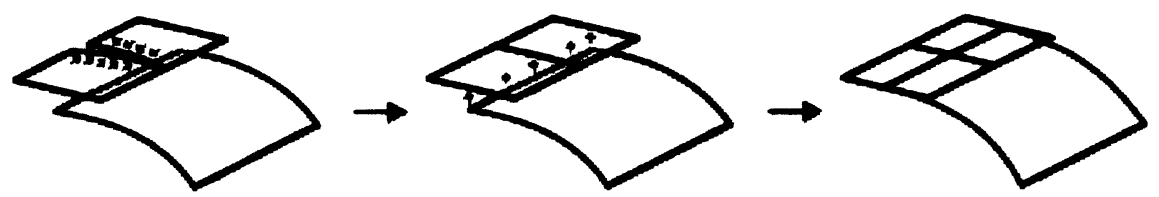

(b) Example of appropriale sequence of global moditication

Fig.4 Difference in the State of Combination by the Sequence of Global Modification

\subsection{Calculation of Surface Intersection of Offset Base Surface}

An AF combination boundary edge is formed through the processing described in the preceding item 3.4. In the case of an offset surface where a mixture of AF combination and convex/concave combination is present, it occurs that the surface intersection cannot be obtained from a generally used calculation of surface intersection or, even if the edge is obtained, it has subtle dislocation from the AF boundary edge, which may fail the boundary loop of a component surface from being closed. This is because the AF combination portion is in the overlapping state and the calculation of surface intersection becomes unstable in the vicinity of this singular point.

This problem is solved by a pincer movement calculation of surface intersection. This method obtains a component point $\mathrm{OP}$ on the intersection of the offset surfaces of OS0 and OS1 from the surface normals N0 and N1 at a component point $\mathrm{P}$ on the intersection of the source surfaces of $\mathrm{S} 0$ and $\mathrm{S} 1$ shown in Fig.5 applying a pincer movement calculation iteratively. This is a robust calculation method of surface intersection proper to the offsetting. 


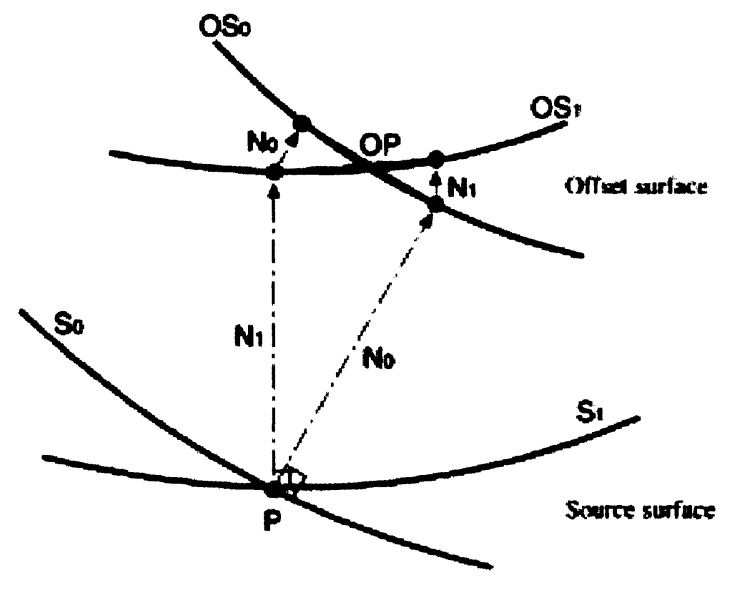

Fig.5 Pincer Movement Calculation Method of Surface Intersection

\subsection{Boundary Path Recognition of the Offset Surfaces}

Topological relation of the AF combined section of the offset surface remains basically unchanged from the source surface (with minor dislocation being absorbed, however). Conversely, topological relation of the convex/concave combinations change and it is difficult for us to foresee the topological relation after the change.

In the first place, a primary loop is formed on the own surface (offset base surface Fig.6(c)(d)) by sequentially calculating the intersection points of the intersection curves between the own surface and its adjacent offset surfaces according to the sequence of the component surface boundary path (Fig.6(b)) as recognized in the preceding item 3.1. Now, if the adjacent surface is either a virtual image surface or reversed surface, it is already deleted. So the intersection with further adjacent surface is obtained and added to the primary boundary loop. If the intersection fails to be obtained notwithstanding the presence of the adjacent surface or if the intersection point of the surface intersections fails to be obtained, the surface intersection with further adjacent surface and its addition to the primary boundary loop are done likewise. Surfaces where the primary boundary loop is not closed should be deleted as the buried surfaces.

Next, detect the intersection on the primary boundary loop of the own surface and separate the loops (Fig.6(e)). As the result, leave those loops having identical direction to the boundary loop of the source surface as the secondary boundary loops and delete all other loops in reverse direction (Fig.6(f)). The secondary boundary loops become the component surface 
boundary loops of the own surface. Here, delete the surface where all the loops are in reverse direction as a reverse surface.

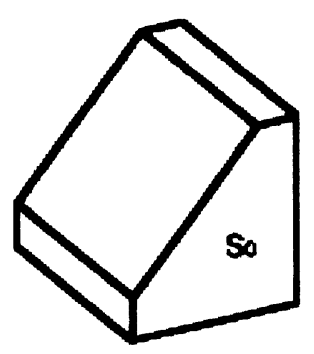

(a) Source composise gurtace modey

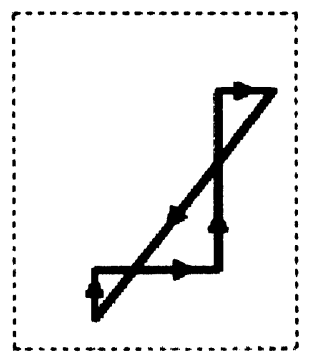

(a) Primary boundery locp

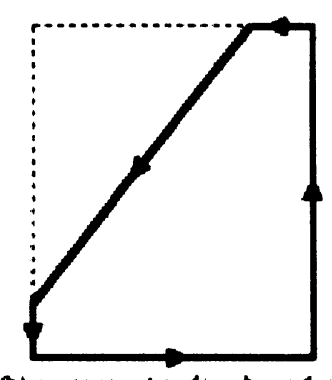

(b) conporent entho boundiny locp (xolid line) of the evures surtice So and the bese surtece boundery (baben lec)

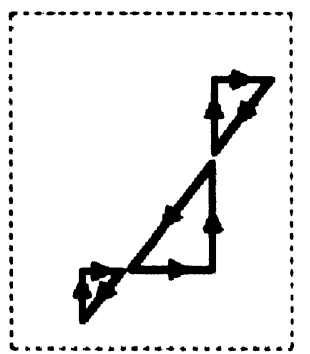

(a) Suparesion of the inverections of uhe poimey boundery loop

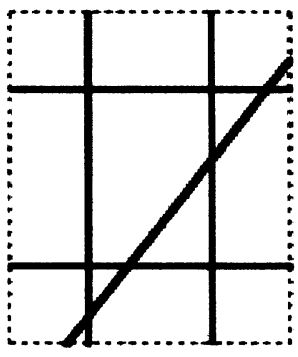

(c) Irveractions of the offent bese surtace

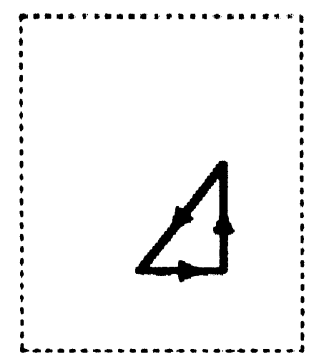

In Secondey boundary locp

Fig.6 Recognition Process of Boundary Path

\section{APPLICATION RESULTS}

Photo.1 shows the result of application of this algorithm to the front bumper. It shows that the offset surfaces vary smoothly without being cracked under the global modification. Moreover, as much as $71 \%$ of the offset surfaces to the source surfaces underwent topological changes, where the boundary path recognition detects the topological change correctly, enabling overall offset of a composite surface.

Fig. 7 shows that the allocated manpower is by far reduced compared to the conventional case. In the present stage, however, approximately $6 \%$ of surfaces to the number of source surfaces fail to be formed. Therefore it is necessary to include the manpower for repairing the failed sections.

Principal causes for the failure are as follows: 


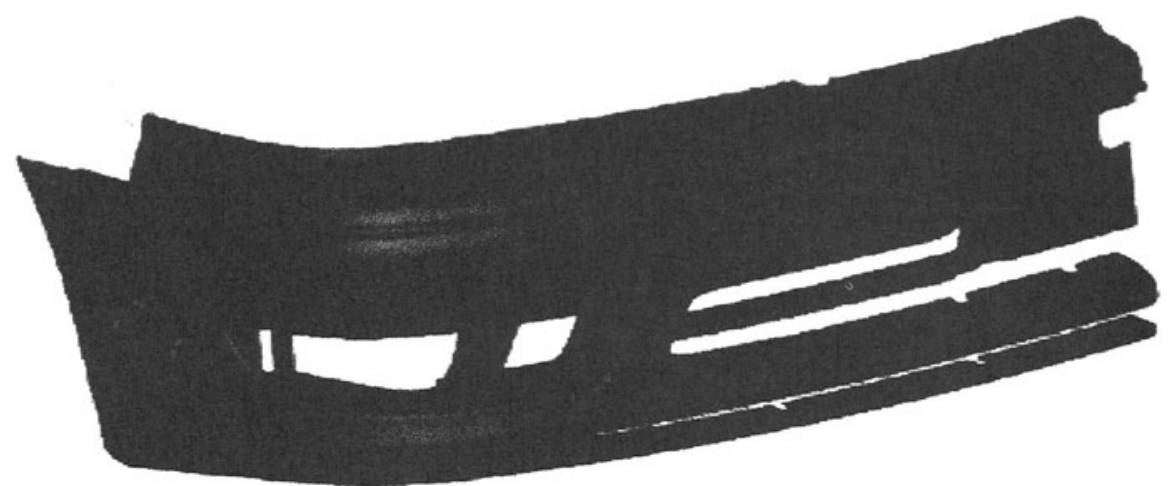

(a) Surface shape (design surface)

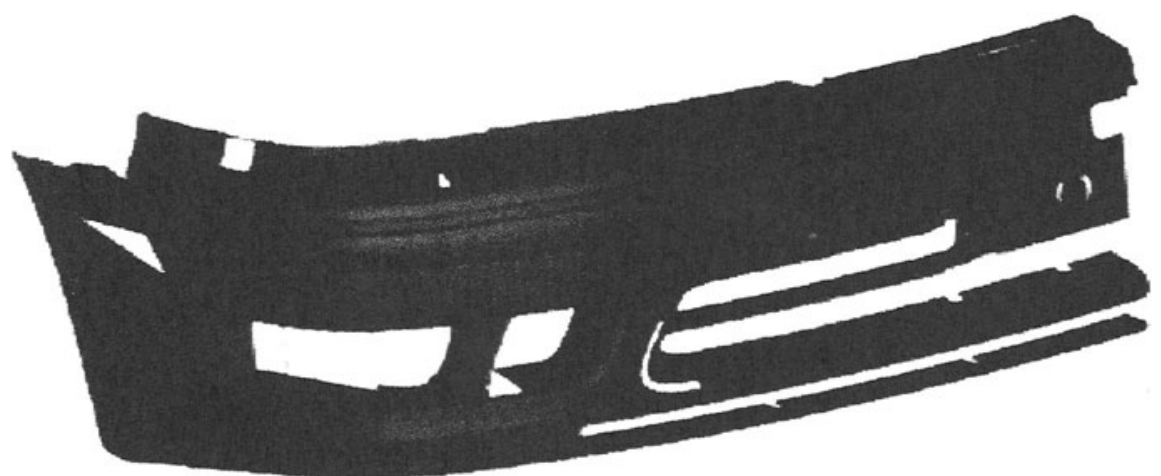

(b) Inner surface shape (overall offset of composite surface) 3.0 to $3.3 \mathrm{~mm}$ variable offset

Photo.1 Application Result to the Front Bumper

(1) Source surfaces are cracked.

(2) Fine-detailed source surfaces cannot be offset.

All of those source surfaces are the component surfaces of some complex sections and these problems cannot be taken merely as the accuracy problems of the source surfaces. In other words, it must be taken up as the problem of the composite surface application technology in the future.

We have reviewed the commercial CAD systems but none of them could make an overall offset of the bumper surfaces successfully. 


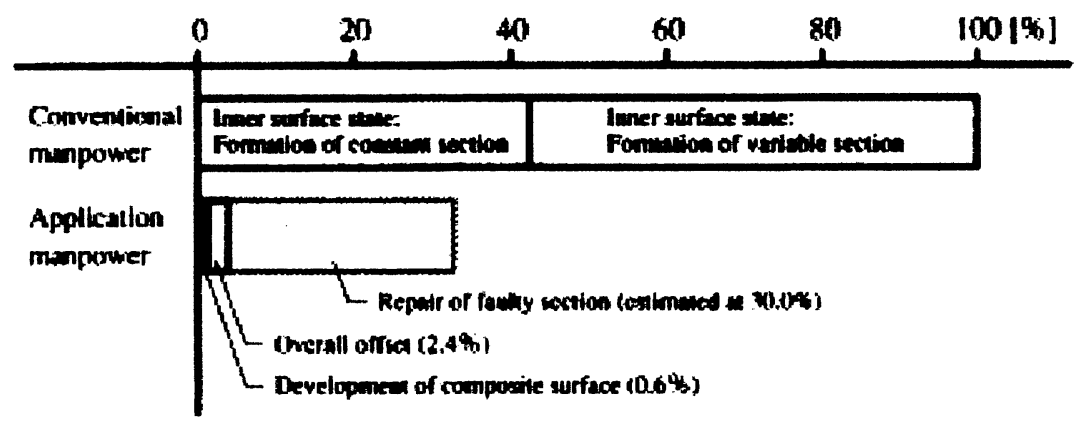

Fig.7 Comparison of Modelling Manpower for Front Bumper

\section{CONCLUSIONS}

We have devised an overall offset algorithm of composite surfaces and started applying it for practical use. We are confident that the algorithm contributes much to the reduction of the surface modelling man-hours and the lead time, which were the initial target for our studies.

We will be grappling with the following problems in the future:

(1) Application of global modification technology to the healing of cracks in the composite surface. (Partial repair needed for the cracks of the source composite surface model or the cracks due to design modification)

(2) Improved quality of variable offset surface. (Elimination of waving in the surface or folding between surfaces)

\section{ACKNOWLEDGEMENTS}

The authors wish to thank members of TOYOTA Soft Engineering, Inc. and IVIS, Inc. for the efforts given in installing the present algorithm for actual application.

\section{REFERENCES}

(1) R.Farouki: Exact offset procedures for simple solids. Computer Aided Geometric Design, Vol. 2, No. 4, P.257-279 (1985)

(2) J.Rossignac, A.Requicha: Offsetting operations in solid modelling. Computer Aided Geometric Design, Vol. 3, No. 2, P.129-148 (1986) 